\title{
Pain Treatment for Older Adults During Prehospital Emergency Care: Variations by Patient Gender and Pain Severity
}

\author{
Timothy F. Platts-Mills ${ }^{\star}, \dagger$, Katherine M. Hunold§, Mark A. Weaver $\ddagger$, Ryan M. Dickey ${ }^{\dagger}$, \\ Antonio R. Fernandez ${ }^{*}$, , Roger B. Fillingim ${ }^{\|, \#}$, Charles B. Cairns ${ }^{*}$, and Samuel A. McLean ${ }^{*} \dagger$ \\ "Department of Emergency Medicine, University of North Carolina, Chapel Hill, North Carolina \\ tDepartment of Anesthesiology, University of North Carolina, Chapel Hill, North Carolina \\ ‡Department of Medicine, University of North Carolina, Chapel Hill, North Carolina \\ $\S$ School of Medicine, University of Virginia, Charlottesville, Virginia \\ "North Florida/South Georgia Veterans Health System, Gainesville, Florida \\ "EMS Performance Improvement Center, Department of Emergency Medicine, University of \\ North Carolina, Chapel Hill, North Carolina
}

\#College of Dentistry, University of Florida, Gainesville, Florida

\begin{abstract}
Older adults are less likely than younger adults to receive analgesic treatment during emergency department visits. Whether older adults are less likely to receive analgesics during protocolized prehospital care is unknown. We analyzed all ambulance transports in 2011 in the state of North Carolina and compared the administration of any analgesic or an opioid among older adults (aged 65 and older) versus adults aged 18 to 64 . Complete data were available for 407,763 transports. Older men were less likely than younger men to receive an analgesic or an opioid regardless of pain severity. Among women with mild or moderate pain, older women were less likely than younger women to receive either form of pain treatment, but among women with more severe pain (pain score 8 or more), older women were more likely than younger women to receive pain treatment. Further, among women with mild or moderate pain, the oldest patients (aged 85 and older) were the least likely to receive any analgesic or an opioid, but among women with severe pain the oldest patients were the most likely to receive treatment. Further research is needed to assess the generalizability of this interaction between age, gender, and pain severity on pain treatment.
\end{abstract}

Perspective-During prehospital care in North Carolina in 2011, older adults were generally less likely to receive pain treatment. However, older women with severe pain were more likely to receive treatment than younger women with severe pain. These results suggest an interaction between age, gender, and pain severity on pain treatment.

\section{Keywords}

Pain; geriatrics; emergency treatment; prehospital 
Older patients with acute pain are less likely to receive pain medication than younger patients during emergency department (ED) care. $8,10,19,20,29$ However, the epidemiology of acute pain treatment for older adults is incompletely understood, as are the causes for observed differences between older and younger adults. ${ }^{7}$ A substantial portion of older ED patients are transported to the ED by ambulance, ${ }^{22,30}$ which provides an early opportunity for pain treatment under different conditions. In contrast to care in the ED, prehospital care is usually only provided to 1 patient at a time and, in the United States, treatments are defined by protocols. The prehospital setting is not only different from the ED but also an important opportunity for pain treatment. Severe pain is common among patients transported by ambulance, and the treatment of pain by emergency medical services (EMS) providers substantially reduces the time to initial treatment. ${ }^{14}$

Adults aged 65 and older account for an estimated 38\% of prehospital transports in the United States, and this number is projected to markedly increase over the next 2 decades. ${ }^{22,26}$ Prior studies of prehospital pain management have not observed lower rates of pain treatment for older adults. For patients receiving prehospital care in Paris in 2007, no association between patient age and the receipt of analgesics was observed. ${ }^{6}$ One Australian study found that older adults were more likely to report a clinically important reduction in pain severity. ${ }^{9}$ Another Australian study found that women were less likely to receive pain medication than men but did not find a difference in pain management between older and younger patients. ${ }^{13}$

The purpose of this descriptive study was to compare analgesia administration for older versus younger adults during ambulance transport across a large and diverse patient population with care provided by EMS providers guided by a pain management protocol. We hypothesized that because of the presence of a standardized treatment protocol that does not differentiate patients according to age, pain management during prehospital care would be similar for older and younger adults.

\section{Methods}

\section{Study Population}

We analyzed data collected by the Emergency Medical Services Performance Improvement Center (EMSPIC), ${ }^{17}$ which captures prehospital care reports corresponding to each ambulance transport in the state of North Carolina. Care within the state is provided by 433 EMS agencies and an estimated 38,200 EMS personnel, but all systems use the same pain management protocol. This protocol recommends administration of oral ibuprofen, acetaminophen, or aspirin for adults with mild pain, and parenteral ketorolac or opioids for patients with moderate or severe pain regardless of age. ${ }^{1}$ This study was determined to be exempt from review by the University of North Carolina at Chapel Hill institutional review board because the analysis uses deidentified data from an administrative database and the potential for deductive disclosure or another form of patient harm is extremely low.

We accessed data on all EMS transports for patients aged 18 years and older in North Carolina during 2011. These data were restricted for the purpose of analysis in the following ways (Fig 1). First, patients were included only if they were transported by EMS (ie, incident disposition of "treated, transported by EMS"). Patients treated on scene and not transported were excluded because in most cases these patients either decline care or are judged to be not sufficiently ill to require EMS transport. Pain medications are not typically administered to these patients. Second, patients were included only if complete data were available for patient age, race, gender, pain severity, and EMS treatment duration. Third, we identified several cases in which the EMS treatment duration was impossibly long (eg, several days). It is our understanding that these apparent errors were the result of the 
incorrect entry of dates for arrival to scene or arrival to hospital. To remove implausible values, we excluded patients with EMS treatment duration longer than 4 hours. Fourth, we excluded patients who had a systolic blood pressure less than $90 \mathrm{~mm} \mathrm{Hg}$ at any time during their transport or for whom systolic blood pressure was unrecorded, because hypotension is listed as a contraindication to analgesic treatment in the state's pain management protocol.

\section{Measures}

All data were recorded by EMS personnel during or immediately after the period of patient care. These data were then captured by the North Carolina EMSPIC either using a webbased application or imported by EMS agencies using commercial vendor software. ${ }^{17}$

Patient age at the time of transport was calculated using patient reported date of birth. For the primary analysis, age was categorized as 18 to 64 (the reference group), 65 to 74,75 to 84 , and 85 years and older. The use of 3 categories to represent older adults was used for the primary analysis because our prior work demonstrated that analgesia treatment varied by age even among older adults. ${ }^{20}$ Gender and race were recorded based on patient self-report. For our analysis, race was coded as white, black, or other. Pain severity was self-reported by patients and was recorded by prehospital providers on a 0 to 10 scale. ${ }^{15}$ In order to examine a more homogeneous subset of patients and to exclude patients with hip fractures, which are more likely to occur in older women and may be particularly likely to receive analgesic treatment, analyses were repeated for patients with either back or abdominal pain. Because of the smaller numbers of patients with these complaints, for this analysis, the numeric pain scale was categorized in the following manner: none (pain score $=0$ ); mild $(1-3)$; moderate (4-6); and severe (7-10). ${ }^{12}$ If patients had more than 1 recorded pain score, then the maximum pain score was used as this typically reflects the patient's pretreatment pain score. EMS treatment duration was defined as the time during which the EMS providers were with the patient, starting with the time of arrival on scene and ending with the time of arrival at the hospital.

The reason for EMS dispatch was coded as trauma, nontrauma, or unknown based on the 39 dispatch complaints recorded by EMS. Dispatch complaints of assault, burn, electrocution, fall, hemorrhage/laceration, industrial accident/inaccessible incident/other, stab/gunshot wound, traffic accident, and traumatic injury were categorized as trauma. All remaining known dispatch complaints were categorized as nontrauma. Dispatch complaints of "not known" or "not recorded" were categorized as unknown.

The 2 outcomes examined were the receipt of any analgesic and the receipt of an opioid analgesic. Analgesic treatment was not differentiated by route of administration, dose, or number of treatments provided. Nitrous oxide is listed in the pain control protocol as a treatment option for adults with moderate or severe pain. However, because treatment with nitrous oxide is not taught or supported by the vast majority of EMS agencies in the state and was recorded in only 4 cases in 2011, nitrous oxide was not classified as an analgesic for the purpose of this analysis.

\section{Statistical Analysis}

Initial analyses compared rates of analgesia administration and presence of inherently painful complaints (abdominal pain, back pain) among patients with missing and nonmissing pain scores. All subsequent analyses were performed for patients with complete data. Patient characteristics are presented for 4 subgroups defined by gender and age (18-64 vs $\Varangle 5$ years). For each of these 4 groups, patient and treatment characteristics (race, pain severity, trauma vs nontrauma, and analgesic received) are presented as percentages. EMS treatment duration is presented using medians and lower and upper quartiles. Model results, 
including $P$ values and confidence intervals, are presented for the purposes of describing our study population only and are not intended for making inferences to any larger patient population. Logistic regression was used to estimate odds ratios, adjusted for race, ${ }^{24}$ dispatch complaint, ${ }^{24}$ and EMS treatment duration, ${ }^{18}$ for receipt of analgesics for patients aged 1) 65 to 74,2) 75 to 84 , and 3) 85 and older, all relative to patients aged 18 to 64 , separately by gender and by pain severity. The estimated odds ratios are presented graphically along with $95 \%$ confidence intervals and with best-fit polynomial lines plotted for each of the 6 age and gender subgroups. In order to more clearly convey the magnitude of the differences between older and younger adults and to facilitate comparisons between men and women, the logistic regression models were refit with older individuals collapsed into a single group of patients aged 65 and older. These revised models were then used to test for interactions between age and pain severity, both separately within each gender and across genders, and to estimate the percentages of older and younger men and women who received analgesics, adjusting for race, dispatch complaint, and EMS treatment duration.

Hip fractures are more common in older women ${ }^{11}$ than older men or younger adults and might be more likely to receive analgesics than other forms of painful conditions. Thus, in order to exclude patients with this confounding condition, we repeated the estimation of odds ratios for receipt of any analgesic or an opioid for older versus younger adults with a restricted sample of patients with abdominal and back pain. These models were also stratified by pain severity and gender and adjusted for race, dispatch complaint category, and EMS treatment duration. All analyses were conducted using SAS 9.2 (SAS Institute Inc, Cary, NC).

\section{Results}

\section{Patient Characteristics}

Of 1,290,209 EMS dispatches in North Carolina in 2011, a transport occurred and complete data were available for 407,763 (Fig 1). Patients for whom pain scores were available were more likely to have received an analgesic than patients with missing pain scores $(6.2 \%$ vs $2.1 \%$ ). Among transports with complete data, $50 \%$ of women were aged 65 or older compared to $40 \%$ of men (Table 1). Adults aged 18 to 64 were more likely to report moderate or severe pain than adults aged 65 or older (among women: $46.9 \%$ vs $23.1 \%, P<$. 001; among men: $42.6 \%$ vs $19.0 \%, P<.001$ ). The majority of transports were for white patients with a nontrauma dispatch complaint. EMS treatment duration was similar for younger and older patients, with median durations of 28 to 31 minutes. The most common category of pain medication administered was an opioid; the most common opioids administered were morphine (2.9\% of all transports with complete data), fentanyl (1.6\%), and hydromorphone $(.8 \%)$.

\section{Odds Ratios of Analgesic Receipt for Transports of Older Versus Younger Patients}

Among women with mild or moderate pain, older patients were generally less likely to receive any analgesic or an opioid than patients aged 18 to 64 (Figs 2 and 3, respectively, top panels). However, among women with severe pain, older patients were more likely to receive any analgesic or an opioid. Further, whereas among women with mild or moderate pain the oldest women (aged 85 and older) had the lowest odds ratios for analgesic and opioid receipt, among women with pain scores of 8 or higher the oldest female patients had the highest odds ratios of analgesic and opioid receipt. Among men, older men were generally less likely to receive any analgesics or an opioid than men aged 18 to 64 for pain scores of 0 to 9 (Figs 2 and 3, bottom panels). Among men with pain scores of 10, the differences in receiving an analgesic or an opioid were less notable. The number of female and male patients in each of these age and pain severity categories is shown in Table 2. 


\section{Interactions Between Age and Pain Severity}

Fig 4 presents model-estimated percentages of patients who received any analgesic by gender, age, and pain severity adjusted for race, EMS dispatch complaint, and treatment duration. Among women with mild or moderate pain, those aged 65 and older were less likely to receive an analgesic. However, among women with severe pain, older women were more likely to receive an analgesic than younger women. A test for an interaction between age and pain severity among women was significant $(P<.001)$. Among men, younger men were nearly twice as likely to receive analgesics for mild pain as older men, but although this ratio significantly decreased for more severe pain (test for interaction $P<.001$ ), the order never reversed as it did among women. These observations suggest that among the study population, pain severity had a greater influence on the relationship between age and pain treatment in women than in men, a hypothesis tested by including a 3-way interaction between gender, age, and pain severity in the model $(P<.001)$. An interaction between gender, age, and pain severity was also observed for the receipt of opioid analgesics. Across all age groups and pain severity scores, women were less likely than men to receive either any analgesic (overall odds ratio for female vs male $=.75,95 \%$ confidence interval $=.73-$. $79)$ or an opioid $(.71, .67-.75)$.

\section{Odds Ratios of Analgesics Receipt Among Patients With Abdominal or Back Pain}

Among the subset of patients reporting abdominal or back pain, an interaction between gender, age, and pain severity was again observed (Table 3). Older men and older women with mild or moderate pain had much lower odds of receiving analgesics or opioids than younger patients, with odds ratios ranging from .29 to .57 . Older female patients with severe abdominal or back pain were still less likely to receive analgesics or opioids than younger patients, but the odds ratios were closer to 1 .

\section{Discussion}

Older adults transported via EMS in the state of North Carolina in 2011 were, in general, less likely than younger adults to receive any analgesic or an opioid analgesic. However, older women with severe pain were more likely to receive pain medication than younger women with severe pain. Differences in pain treatment between older and younger adults were most pronounced for the very oldest patients; women aged 85 or older with mild or moderate pain had the lowest odds ratios for pain treatment as compared to women aged 18 to 64 , but women aged 85 or older with severe pain had the highest odds ratios for treatment. This interaction between pain severity and age was present in men to a much lesser degree; only the very oldest men with a pain score of 10 were more likely than patients aged 18 to 64 to receive analgesics or opioids. The findings from this exploratory analysis indicate an interaction between patient gender, age, and pain severity on the management of acute pain that has not previously been described in either a prehospital or ED setting.

We did not anticipate that the relationship between age and pain treatment would differ either by pain severity or by gender. As a result, additional work is needed to assess their generalizability both across time and across acute care settings. Nonetheless, odds ratios for pain treatment for older versus younger women increased progressively with increasing pain severity and the difference in pain treatment rates for older versus younger patients was consistently most pronounced for the oldest patients. These findings in a study population of more than 400,000 patients suggest that the observed interaction may be a phenomenon that warrants further study.

The finding of higher rates of pain treatment in older women with severe pain than younger women with severe pain is contrary to the lower rates of acute pain treatment among older 
adults reported in several other studies. ${ }^{6,8,10,19,29}$ Several factors might contribute to our findings. The generally lower rates of treatment in older adults may be due to concerns about side effects, ${ }^{2,5,23}$ ageism, or older adults' declining pain treatment. Even though effective treatment of pain does not alter the clinical evaluation of ED patients, ${ }^{25}$ concern about masking a diagnosis may be a reason for some EMS providers to withhold treatment. The higher rates of treatment in older women with severe pain might be due to a threshold effect, whereby for older women with mild or moderate pain the risk of side effects are judged by EMS providers to outweigh the benefits of treatment, but for older women with severe pain the benefits of treatment are judged to outweigh the risks. Further, because severe pain was relatively rare in older women $(12.8 \%)$ but more common in younger women (31.1\%), EMS providers may be more likely to believe and want to treat severe pain in older women. Conversely, EMS providers may be somewhat dismissive of severe pain in younger women. An alternative explanation may be that older women are more likely to decline pain treatment than younger women when the pain is mild or moderate, but more likely to request pain treatment when the pain is severe.

Even in a setting in which care is guided by a protocol that does not differentiate by age or gender, we observed substantial variance in care based on these patient characteristics. The variance in care for pain management by age and gender in this study and the overall low rate of analgesia administration is contrary to the high rate of protocol compliance for interventions such as cervical-spine clearance ${ }^{28}$ and care for patients with atraumatic chest pain. ${ }^{3}$ The variance in care and the low rate of analgesia treatment seen in this and other prehospital pain studies ${ }^{6,13}$ suggests that many EMS providers are not informed by their medical director of the importance of analgesia administration. Several other possible reasons for the low rate of opioid administration among patients with severe pain may have affected the observed rates: extra effort required of EMS providers who administer opioids, including additional paperwork and obtaining a witness for disposal of unused medication; absence of EMS providers with paramedic-level training (required to administer opioids) for some transports; or difficulty obtaining intravenous access.

We found that women were less likely to receive analgesia than men, especially among individuals aged 18 to 64 with moderate or severe pain. For this group, the absolute differences in pain treatment ranged from 2 to $6 \%$ and the relative differences ranged from 27 to $41 \%$. The lower treatment rate for women than men is of particular concern given the evidence for the increased susceptibility of women to both acute and chronic pain conditions. ${ }^{4}$

There are several limitations to this study. The data received from prehospital care reports do not include information regarding potential confounders such as the gender and experience of EMS providers, patient socioeconomic status, ${ }^{21}$ or patient preferences for treatment. Concordance of patient and provider gender has been shown to be associated with increased analgesia treatment in the $\mathrm{ED},{ }^{27}$ and it is possible that gender concordance also influences pain management decisions in the prehospital setting. We did not distinguish between types of opioids or nonopioids, the dosage or number of doses of medications given, or the effect of treatment on pain scores. This study describes prehospital analgesia treatment for the state of North Carolina in 2011, which has a single pain management protocol for the entire state. Practice patterns may be different in other settings. Pain scores were missing for more than half the study population, which is similar to national estimates of missing pain documentation in EMS systems ${ }^{16}$ but nonetheless has the potential to introduce bias. Although patients who were missing pain scores were less likely to receive analgesics than those for whom pain scores were recorded, there were some patients with no recorded pain score who received analgesics. The effect of excluding patients with missing pain scores on the results is unknown. 
In summary, we observe that for prehospital transports in North Carolina in 2011, older patients were less likely to receive pain treatment than younger patients except for older women with severe pain, who were more likely to receive pain treatment than younger women. Further research is needed to assess the generalizability, contributing factors, and consequences of this interaction between age, gender, and pain severity on analgesic treatment.

\section{Acknowledgments}

T.F.P.-M. is funded by a National Institutes of Health (NIH) grant KL2TR000084. M.A.W. is funded by NIH grant UL1TR000083. S.A.M. is funded by NIH grant R01AR056328. The content is solely the responsibility of the authors and does not necessarily represent the official views of the NIH. Data were provided by the North Carolina Office of EMS (NC OEMS) and the North Carolina EMS (NC EMS) Data System. The NC OEMS and the NC EMS Data System supports state, regional, and local EMS and health care-related service delivery from a patient care, resource allocation, and regulatory perspective. This manuscript has been reviewed by NC EMS Data System investigators for scientific content and consistency of data interpretation with previous NC EMS Data System publications, and significant comments have been incorporated prior to submission for publication.

\section{References}

1. Cepeda MS, Farrar JT, Baumgarten M, Boston R, Carr DB, Strom BL. Side effects of opioids during short-term administration: Effect of age, gender, and race. Clin Pharmacol Ther. 2003; 74:102-112.

2. Colwell C, Mehler P, Harper J, Cassell L, Vazquez J, Sabel A. Measuring quality in the prehospital care of chest pain patients. Prehosp Emerg Care. 2009; 13:237-240.

3. Fillingim RB, King CD, Ribeiro-Dasilva MC, Rahim-Williams B, Riley JL 3rd. Sex, gender, and pain: A review of recent clinical and experimental findings. J Pain. 2009; 10:447-485.

4. Furlan AD, Sandoval JA, Mailis-Gagnon A, Tunks E. Opioids for chronic noncancer pain: A metaanalysis of effectiveness and side effects. CMAJ. 2006; 174:1589-1594.

5. Galinski M, Ruscev M, Gonzalez G, Kavas J, Ameur L, Biens D, Lapostolle F, Adnet F. Prevalence and management of acute pain in prehospital emergency medicine. Prehosp Emerg Care. 2010; 14:334-339. [PubMed: 20507221]

6. Green SM. There is oligo-evidence for oligoanalgesia. Ann Emerg Med. 2012; 60:212-214.

7. Hwang U, Richardson LD, Harris B, Morrison RS. The quality of emergency department pain care for older adult patients. J Am Geriatr Soc. 2010; 58:2122-2128.

8. Jennings PA, Cameron P, Bernard S. Determinants of clinically important pain severity reduction in the prehospital setting. Emerg Med J. 2012; 29:333-334. [PubMed: 21252175]

9. Jones JS, Johnson K, McNinch M. Age as a risk factor for inadequate emergency department analgesia. Am J Emerg Med. 1996; 14:157-160.

10. Kannus P, Parkkari J, Sievanen H, Heinonen A, Vuori I, Jarvinen M. Epidemiology of hip fractures. Bone. 1996; 18:57S-63S.

11. Krebs EE, Carey TS, Weinberger M. Accuracy of the pain numeric rating scale as a screening test in primary care. J Gen Intern Med. 2007; 22:1453-1458.

12. Lord B, Cui J, Kelly AM. The impact of patient sex on paramedic pain management in the prehospital setting. Am J Emerg Med. 2009; 27:525-529.

13. McEachin CC, McDermott JT, Swor R. Few emergency medical services patients with lowerextremity fractures receive prehospital analgesia. Prehosp Emerg Care. 2002; 6:406-410.

14. McLean SA, Domeier RM, DeVore HK, Hill EM, Maio RF, Frederiksen SM. The feasibility of pain assessment in the prehospital setting. Prehosp Emerg Care. 2004; 8:155-161.

15. McLean SA, Maio RF, Domeier RM. The epidemiology of pain in the prehospital setting. Prehosp Emerg Care. 2002; 6:402-405.

16. Mears GD, Pratt D, Glickman SW, Brice JH, Glickman LT, Cabanas JG, Cairns CB. The North Carolina EMS Data System: A comprehensive integrated emergency medical services quality improvement program. Prehosp Emerg Care. 2010; 14:85-94. [PubMed: 19947872] 
17. Michael GE, Sporer KA, Youngblood GM. Women are less likely than men to receive prehospital analgesia for isolated extremity injuries. Am J Emerg Med. 2007; 25:901-906.

18. North Carolina Office of EMS 2012 Treatment Protocols

19. O'Connor AB, Zwemer FL, Hays DP, Feng C. Outcomes after intravenous opioids in emergency patients: A prospective cohort analysis. Acad Emerg Med. 2009; 16:477-487.

20. Platts-Mills TF, Esserman DA, Brown DL, Bortsov AV, Sloane PD, McLean SA. Older US emergency department patients are less likely to receive pain medication than younger patients: Results from a national survey. Ann Emerg Med. 2012; 60:199-206.

21. Platts-Mills TF, Hunold KM, Bortsov AV, Soward AC, Peak DA, Jones JS, Swor RA, Lee DC, Domeier RM, Hendry PL, Rathlev NK, McLean SA. More educated emergency department patients are less likely to receive opioids for acute pain. Pain. 2012; 153:967-973. [PubMed: 22386895]

22. Platts-Mills TF, Leacock B, Cabanas JG, Shofer FS, McLean SA. Emergency medical services use by the elderly: Analysis of a statewide database. Prehosp Emerg Care. 2010; 14:329-333.

23. Platts-Mills TF, Richmond NL, Hunold KM, Bowling CB. Life-threatening hyperkalemia following two days of ibuprofen. Am J Emerg Med. 2013; 31:465.

24. Pletcher MJ, Kertesz SG, Kohn MA, Gonzales R. Trends in opioid prescribing by race/ethnicity for patients seeking care in US emergency departments. JAMA. 2008; 299:70-78.

25. Ranji SR, Goldman LE, Simel DL, Shojania KG. Do opiates affect the clinical evaluation of patients with acute abdominal pain? JAMA. 2006; 296:1764-1774.

26. Roberts DC, McKay MP, Shaffer A. Increasing rates of emergency department visits for elderly patients in the United States, 1993 to 2003. Ann Emerg Med. 2008; 51:769-774. [PubMed: 18069088]

27. Safdar B, Heins A, Homel P, Miner J, Neighbor M, DeSandre P, Todd KH. Impact of physician and patient gender on pain management in the emergency department-A multicenter study. Pain Med. 2009; 10:364-372. [PubMed: 18992042]

28. Sebastian RJ, Miller K, Langdorf MI, Johnson D. EMS adherence to a pre-hospital cervical spine clearance protocol. Cal J Emerg Med. 2001; 2:44-46.

29. Terrell KM, Hui SL, Castelluccio P, Kroenke K, McGrath RB, Miller DK. Analgesic prescribing for patients who are discharged from an emergency department. Pain Med. 2010; 11:1072-1077.

30. Wofford JL, Moran WP, Heuser MD, Schwartz E, Velez R, Mittelmark MB. Emergency medical transport of the elderly: A population-based study. Am J Emerg Med. 1995; 13:297-300. 


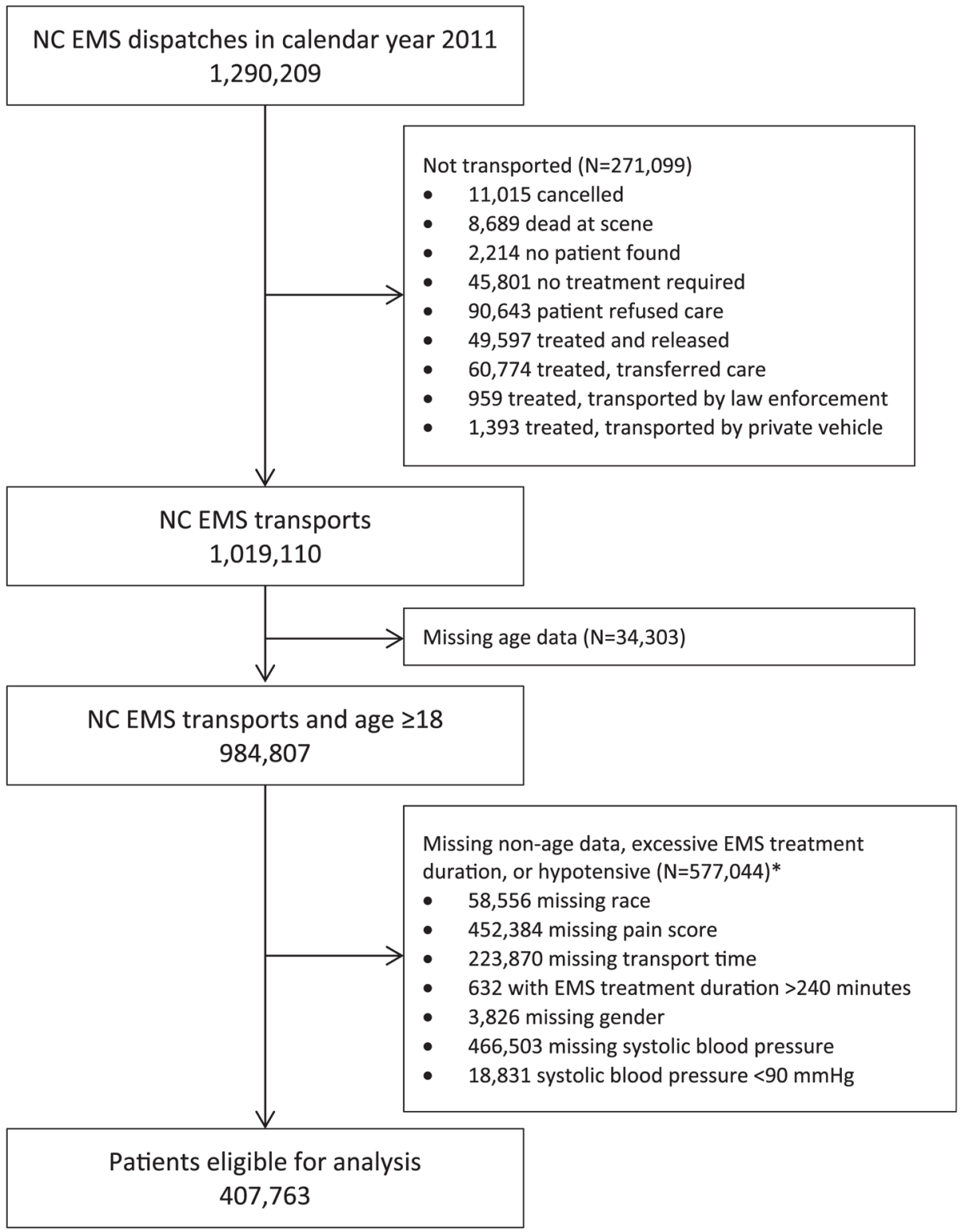

* Categories are not mutually exclusive

Figure 1.

Flow diagram of patients eligible for analysis. 

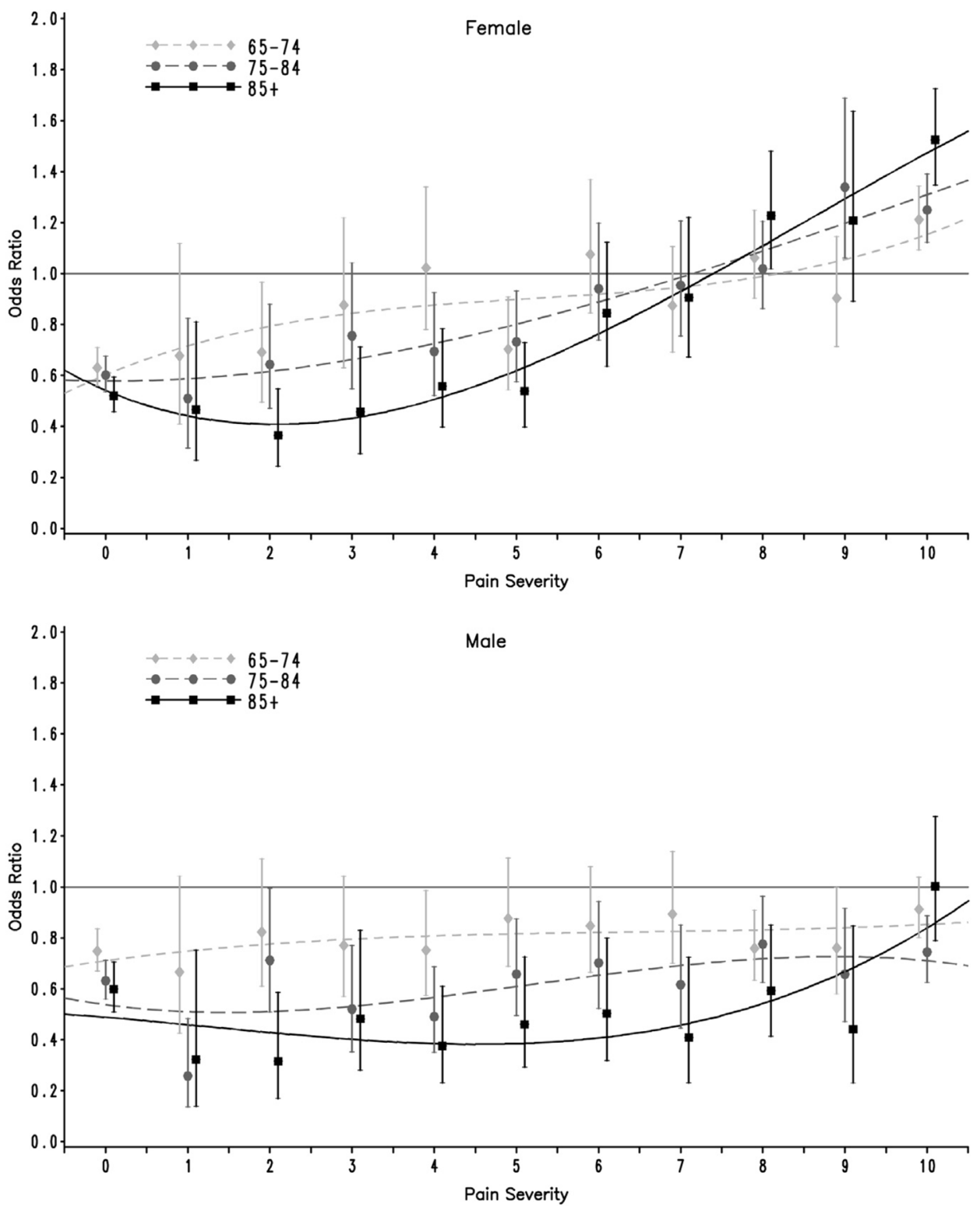

Figure 2.

Odds ratios of older adults versus adults aged 18 to 64 receiving pain medication by pain severity score, stratified by gender and adjusted for race, dispatch complaint, and transport time. Best-fit polynomial lines are shown for each age group. 

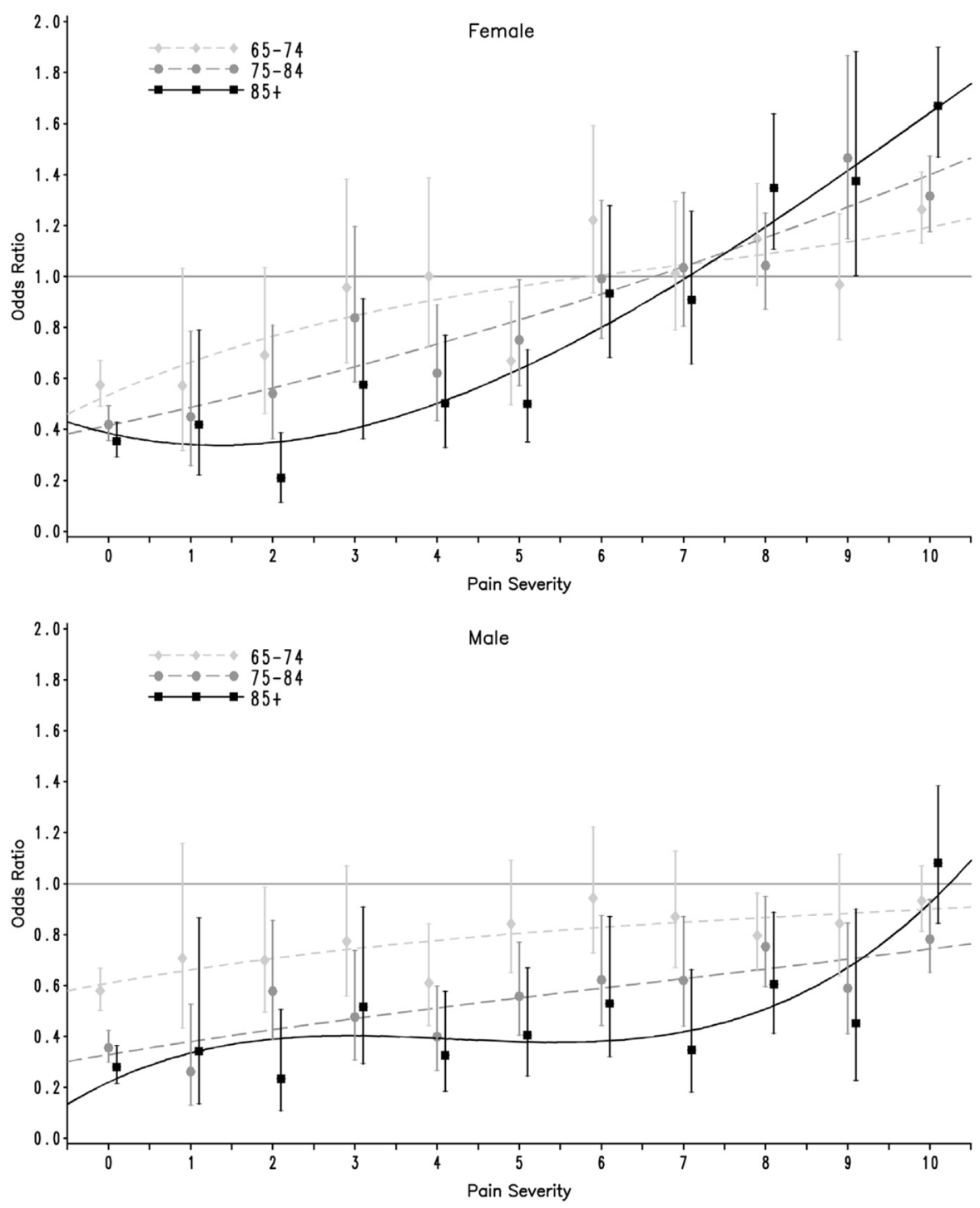

Figure 3.

Odds ratios of older adults versus adults aged 18 to 64 receiving opioid pain medication by pain severity score, stratified by gender and adjusted for race, dispatch complaint, and transport time. Best-fit polynomial lines are shown for each age group. 

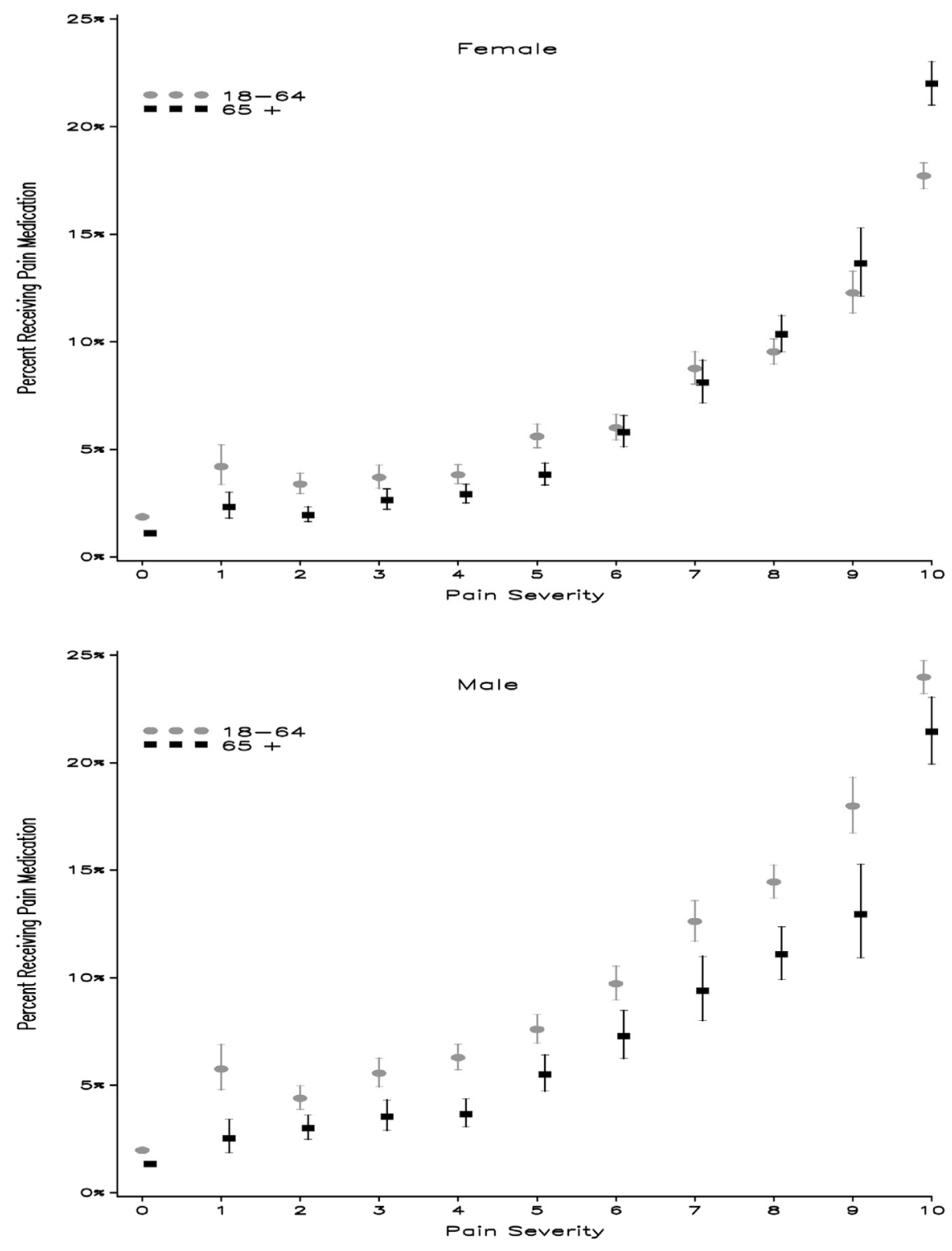

Figure 4.

Model-estimated percentages of patients receiving pain medication by pain severity score, stratified by gender and adjusted for race, dispatch complaint, and EMS treatment duration. 
Table 1

Characteristics of 407,763 Patients With Complete Data Transported by Ambulance in North Carolina in 2011

\begin{tabular}{|c|c|c|c|c|}
\hline \multirow[b]{2}{*}{ Characteristic } & \multicolumn{2}{|c|}{ Women } & \multicolumn{2}{|c|}{ Men } \\
\hline & $18-64(N=115,312)$ & $\searrow 65(N=115,928)$ & $18-64(N=105,675)$ & $\searrow 65(N=70,848)$ \\
\hline \multicolumn{5}{|l|}{ Race $(\%)$} \\
\hline White & 58.1 & 73.4 & 60.3 & 74.5 \\
\hline Black & 37.6 & 24.8 & 35.1 & 23.8 \\
\hline Other & 4.3 & 1.7 & 4.6 & 1.8 \\
\hline \multicolumn{5}{|l|}{ Pain severity (\%) } \\
\hline None $(0)$ & 44.0 & 68.3 & 48.0 & 73.0 \\
\hline Mild (1-3) & 9.2 & 8.9 & 9.43 & 8.0 \\
\hline Moderate (4-6) & 15.8 & 10.3 & 15.3 & 9.1 \\
\hline Severe $(7-10)$ & 31.1 & 12.8 & 27.3 & 9.9 \\
\hline \multicolumn{5}{|l|}{ Dispatch complaint (\%) } \\
\hline Trauma & 16.8 & 14.8 & 19.6 & 11.6 \\
\hline Nontrauma & 69.2 & 71.8 & 66.1 & 75.2 \\
\hline Unknown & 14.1 & 13.4 & 14.4 & 13.2 \\
\hline EMS treatment duration, $\min { }^{*}$ & $28.0(20.0-38.0)$ & $29.0(20.3-40.0)$ & $28.0(20.0-40.0)$ & $31.0(22.0-43.0)$ \\
\hline \multicolumn{5}{|l|}{ Analgesic received $(\%)$} \\
\hline Acetaminophen & .8 & .7 & .7 & .9 \\
\hline Opioid & 5.4 & 3.4 & 7.9 & 3.2 \\
\hline NSAIDs & 1.0 & .3 & 1.0 & .3 \\
\hline Other & 0 & 0 & 0 & 0 \\
\hline Any & 6.9 & 4.3 & 9.2 & 4.4 \\
\hline
\end{tabular}




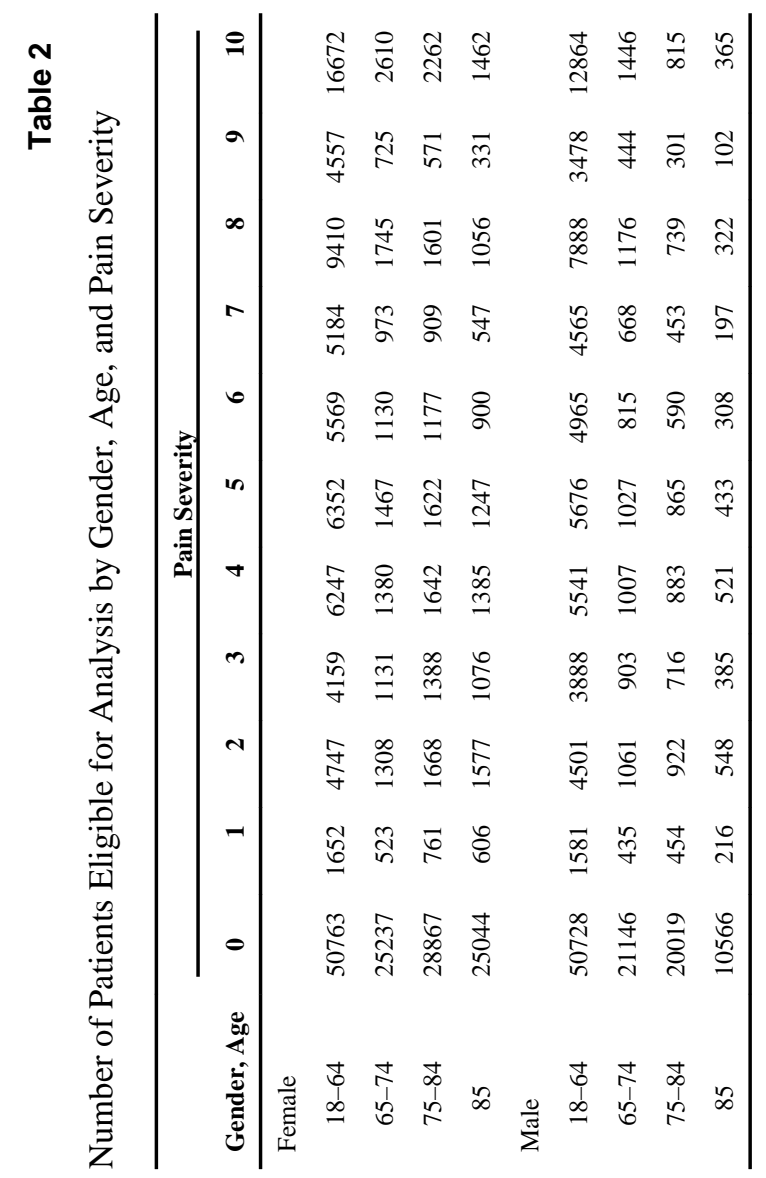




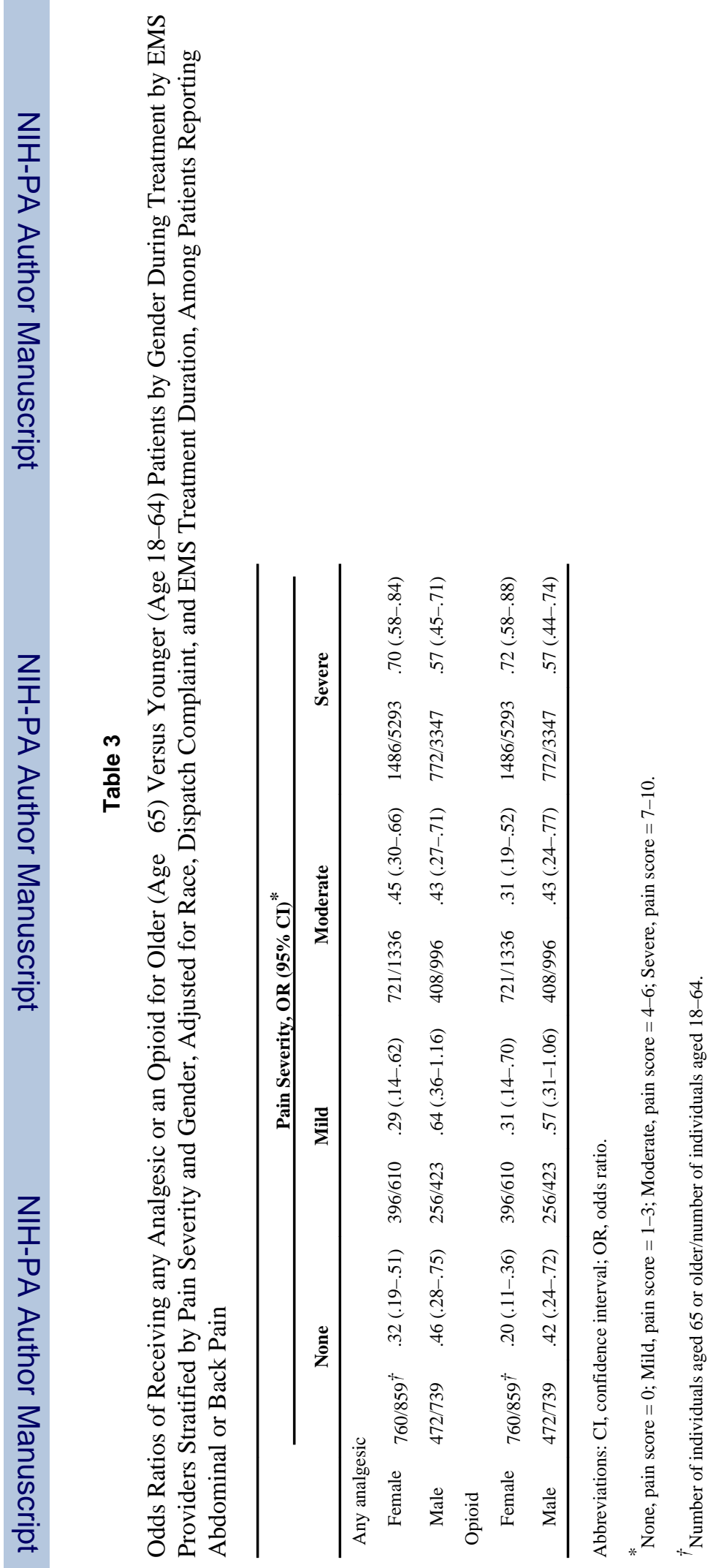

J Pain. Author manuscript; available in PMC 2014 September 01. 